\title{
2.5D virtual reality visualisation of shearography strain data from a canvas painting
}

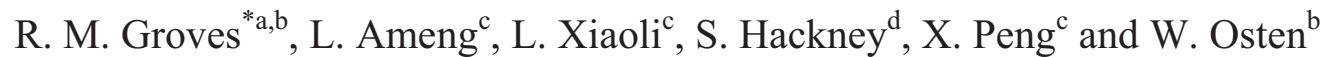 \\ ${ }^{\mathrm{a}}$ Faculty of Aerospace Engineering, Delft University of Technology, \\ Kluyverweg 1, 2600 GB Delft, The Netherlands; \\ ${ }^{b}$ ITO Institut für Technische Optik, Universität Stuttgart, \\ Pfaffenwaldring 9, 70569 Stuttgart, Germany; \\ ${ }^{\mathrm{c} C}$ College of Optoelectronic Engineering, Shenzhen University, 518060 Shenzhen, China; \\ ${ }^{\mathrm{d}}$ Conservation Department, Tate, Millbank, London SW1P 4RG, UK
}

\begin{abstract}
Advanced measurement techniques for the structural diagnostics of artwork are increasingly providing more complex data that needs to be conveyed to conservators in a meaningful way. Holography and speckle interferometry based sensors are commonly used for this application and of these shearography is quite suitable for measurements outside the optics laboratory, due to the stability of using a common path interferometer configuration. Shearography provides noncontact full-field displacement gradient data on surface and sub-surface defects in the form of phase maps. The display of this data in the form of wrapped phase maps is only suitable for experienced users. A further image processing step generates unwrapped phase maps, which in an engineering environment are generally colour coded for display. For artwork measurement applications, the colour variation of the painting itself is important reference for the conservator to locate defect locations. In this manuscript the displacement gradient data is presented as false height on the flat painting surface. A virtual reality viewer, freely downloadable from the internet, is used to display the data and allow the user to interact with it by rotating the object in virtual space. The effect is rather similar to viewing a raked light photograph, however with the advantage of remote or online viewing.
\end{abstract}

Keywords: virtual reality, shearography, structural diagnostics, artwork

\section{INTRODUCTION}

Advanced measurement techniques are increasingly being applied to cultural heritage objects [1] and optical techniques are particularly important as they are often non-contact, non-destructive and can provide high accuracy measurements [2]. The information provided supports many museum activities, including art history research, authenticity, monitoring of the museum environment, loans and transportation, and for conservation. This data needs to be presented to the user in a meaningful way and there are many possibilities to present these datasets. Tables of data are often difficult to interpret, so graphical techniques such as line and surface plots, colour-coded maps and videos are commonly used. Virtual reality techniques are of increasing importance in many fields, including mass media, manufacturing and medicine. In this paper the authors investigate the application of virtual reality for the display of shearography data from a canvas painting.

Shearography is a speckle interferometry technique, closely related to holography, that measures full-field displacement gradient. It is sensitive to small surface and sub-surface changes that occur when an object is loaded and provides data in the form of phase maps. A typical measurement procedure consists of illuminating the artwork using an expanded laser beam, optically mixing the scattered light in a shearing interferometer and recording the resulting reference interferogram using a CCD camera. The procedure is repeated after the object is loaded and the interferograms are correlated in a computer to generate the displacement gradient maps. Because of the interferometric sensitivity of shearography, artwork can be measured non-destructively by applying small temperature changes $\left(1-2{ }^{\circ} \mathrm{C}\right)$.

*r.m.groves@tudelft.nl; phone +31 1527 88230; fax +31 1527 81151; www.tudelft.nl

O3A: Optics for Arts, Architecture, and Archaeology II, edited by Luca Pezzati, Renzo Salimbeni,

Proc. of SPIE Vol. 7391, 739109 - (c) 2009 SPIE · CCC code: 0277-786X/09/\$18 · doi: 10.1117/12.827519

Proc. of SPIE Vol. 7391 739109-1 
Virtual reality (VR) technology can provide a powerful new tool for the cultural heritage community. It is a technique which enables complex data sets to be visualized interactively in a 3d-reality controlled by the user. Measured data sets are fused with a $3 \mathrm{~d}$ model of the object using dedicated algorithms and software and can be viewed using freely downloadable VR viewers. Most current VR environments are primarily visual experiences, displayed either on a computer screen or through special or stereoscopic displays.

By combining the philosophy of 3D digitizing and shearography we can explore a new multimodal imaging modality that can measure and evaluate physical parameters of artwork like $2.5 \mathrm{D}$ or $3 \mathrm{D}$ shape, deformation, micro-displacement, strain map, color texture, etc. The manuscript contains the full implementation details for a shearography measurement on a sample artwork and the preparation of the associated VR file. Supporting virtual reality files and instructions are available at [3].

\section{SHEAROGRAPHY}

\subsection{Theory}

Shearography, also known as speckle shearing interferometry, [4] is a full-field speckle interferometry technique sensitive to displacement gradient. A typical configuration is shown in Figure 1. The object under investigation is illuminated using the expanded beam from a long coherence length laser. For an optically rough object surface this forms a speckle pattern. This speckle pattern is optically mixed in the shearing interferometer, a process which converts phase information to amplitude, forming an interferogram. This reference interferogram is recorded using a CCD camera. A commonly used shearing device is a shearing Michelson interferometer, which contains a beamsplitter, a reference mirror and a shearing mirror, which is usually tilted horizontally or vertically to apply the shear. To determine the displacement gradient change after applying a loading to the object, a second interferogram is recorded after loading and from these two interferograms the displacement gradient can be calculated by the computer.

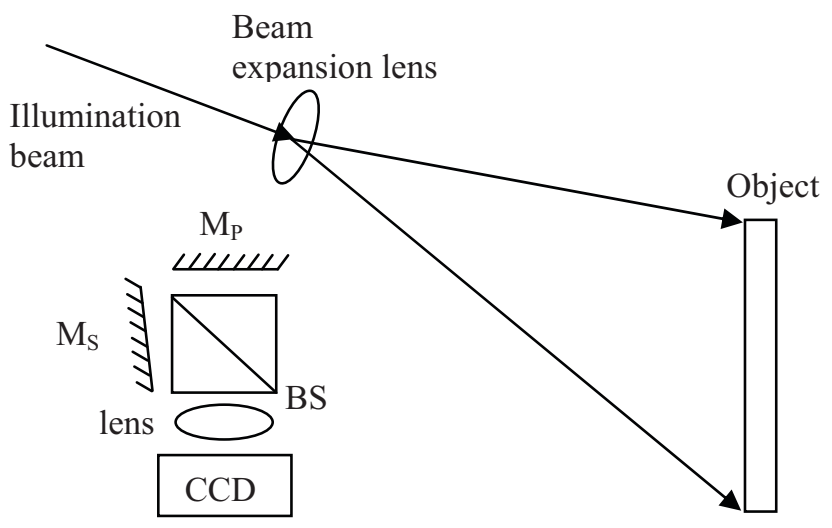

Figure 1 shows a typical shearography experimental layout. The illumination beam is expanded by a lens to illuminate the surface of the object. Light scattered by the object is optically mixed in the interferometer and the resulting interferogram is imaged onto the camera chip, $C C D$, by a lens. The interferometer components are beamsplitter, $B S$, phase shifting mirror, $M_{P}$, and shearing mirror, $M_{S}$.

The component of displacement gradient measured is determined by the illumination and viewing directions and the direction of shear applied in the interferometer. The sensitivity is controlled by the amount of shear applied. The most common shearography configuration measures out-of-plane displacement gradients, as given by Equation 1:

$$
\Delta \phi_{(x, y)}=\frac{4 \pi}{\lambda} \frac{\delta w}{\delta x}(x, y) \text {, or } \Delta \phi_{(x, y)}=\frac{4 \pi}{\lambda} \frac{\delta w}{\delta y_{(x, y)}} d y
$$

where $\Delta \varphi$ is the phase change in the interferometer, $\lambda$ is the optical wavelength, $\delta w / \delta x$ is the out-of-plane displacement differentiated in the horizontal direction, $\delta w / \delta y$ is the out-of-plane displacement differentiated in the vertical direction, and $d x$ and $d y$ are the horizontal and vertical shear magnitudes, respectively. 
Advanced phase and image processing techniques are used to calculate the displacement gradient from the interferograms. In the phase-shifting technique, wrapped phase maps are determined, showing the direction of displacement gradient in addition to its magnitude [5]. These wrapped phase maps can be further processed by unwrapping [6] and rescaling to determine the displacement gradient map. Displacement gradient maps are generally preferred by the instrument user, even though the palate folding effect of wrapping the phase maps can sometimes be an advantage for defect detection.

\subsection{Instrumentation}

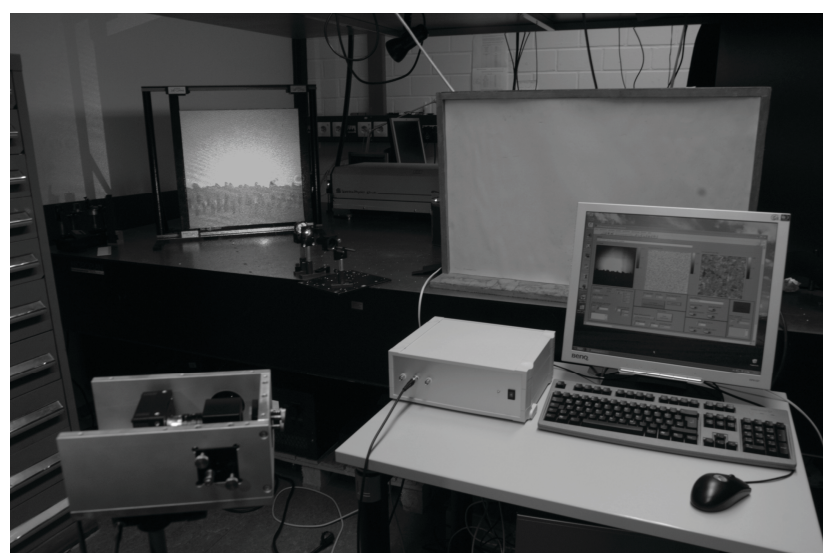

(a)

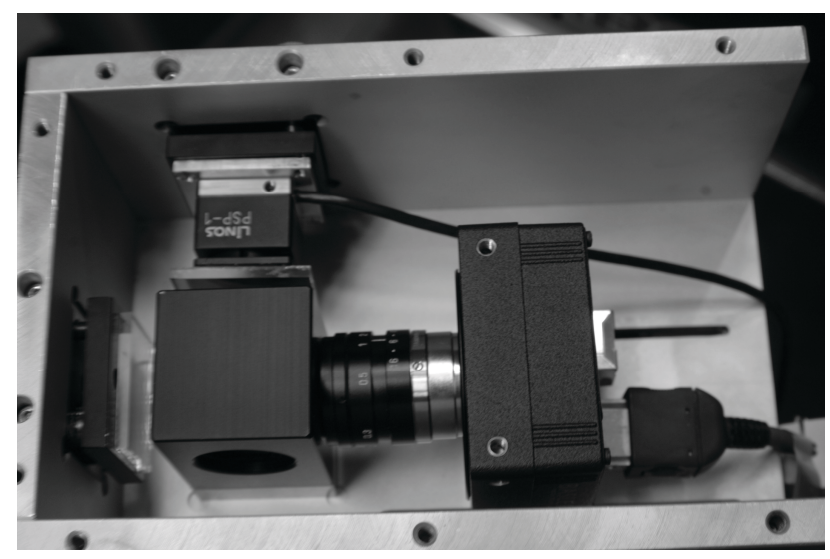

(b)

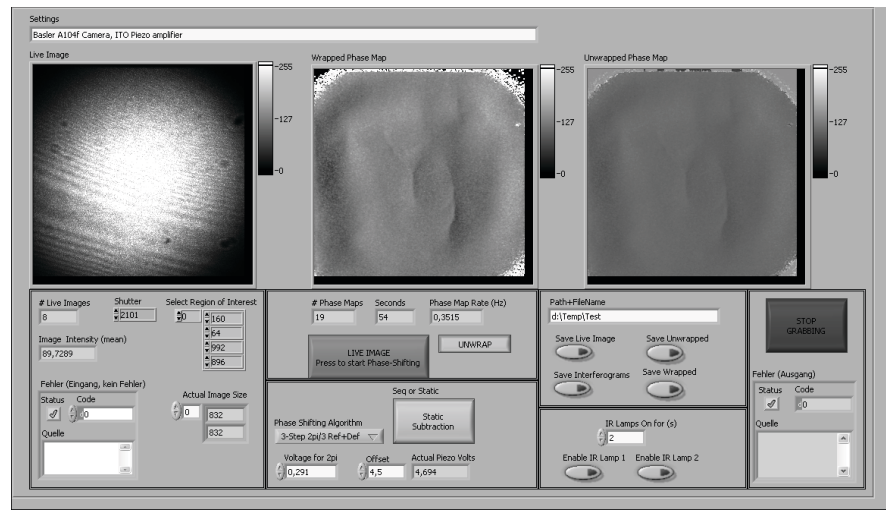

(c)

Figure 2(a) a photograph of the shearography measurement setup, (b) a close-up of the interferometer head composed of shearing Michelson interferometer, lens and camera and (c) the user interface for the software showing from left to right live display, wrapped phase map and unwrapped phase map.

The shearography instrumentation follows the experimental configuration shown in Figure 1. The object is illuminated by the expanded beam from a Coherent Verdi-V10 laser (532 nm, optical power adjustable from $\sim 50 \mathrm{~mW}$ to $10 \mathrm{~W})$. A typical optical power used for the artwork samples described in this paper would be $500 \mathrm{~mW}$. The optical head consists of a shearing Michelson interferometer, camera lens (25 mm focal length) and a Basler A102f CCD camera (1392 x 1040 pixels, pixel size $6.45 \times 6.45 \mu \mathrm{m}^{2}$, Firewire interface). The shearing interferometer is composed of a broadband beamsplitter cube, shearing mirror, which can be tilted horizontally or vertically, and a phase-shifting mirror which is controlled by a Linos PSP-1 piezo-electric element. The optical head is interfaced to the PC via an electronics control box which contains the PZT amplifier, Firewire power supply and a USB DAC card (National instruments NI-6008). One Firewire cable and one USB cable connect directly to the PC. The instrument is operated using software developed 
using Labview software (National Instruments) and controls instrument setting, phase-shifting and image capture and performs image processing and live display of phase maps and data archiving. Once the measurement procedure is started the software records reference interferograms, applies a controlled loading to the painting using infra-red lamps and before recording further interferograms. Wrapped and unwrapped phase maps are displayed in real-time and the phase maps are archived to the hard disk. A typical measurement time is $300 \mathrm{~s}$, with interferograms recorded typically every $10 \mathrm{~s}$. Figure 2 shows the experimental configuration and the software user interface.

\section{VIRTUAL REALITY}

Virtual reality is a technique for displaying 3D data in an interface controlled by the user. The $2.5 \mathrm{D}$ virtual reality techniques described here involve converting a data channel - in this case a displacement gradient map - to appear as false height at the object surface. This is of particular importance for artwork, as the usual colour mapping of data techniques used in engineering are less suited to artwork, as the colour of the artwork is an important property of the object for the conservator during his/her work. 2.5D visualization avoids overwriting object colours as the defect map is mapped as a false height perpendicular to the local object surface.

For many objects the first stage of the procedure is to determine the object shape. In recent years, optical 3D measurement is becoming increasingly important and has a wide range of applications, including reverse engineering, rapid prototyping and robot navigation, in addition to virtual reality. The existing 3D measurement techniques may be summarized as stereo vision [7] and structured lighting or laser scanning [8]. This second group includes coded lighting patterns, such as binary codes, gray code with phase shifting, De Bruijin sequences, color codes, point-arrays, etc. [9]. Objects with a single flat surface, such as a painting, can be effectively implemented as a planar surface in the VR model.

The steps for the calculation of a virtual reality model for a planar object are shown in Figure 3.

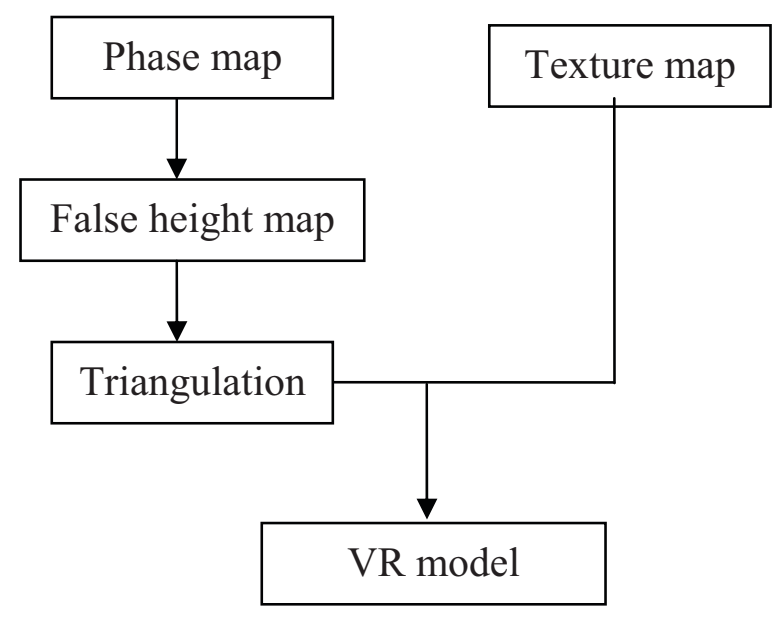

Figure 3 Flow chart of texture-mapping for shearography

The first input parameter is the shearography displacement gradient data. The rows and columns in the data correspond to the pixels of camera sensor of the shearography system, and the pixel's grey value indicates the displacement gradient data. Calibrated system parameters are required to convert displacement gradient (or unwrapped phase) data into a 3D mesh. The accuracy requirements for these parameters are satisfied if the virtual reality model is convincing for the user. This $3 \mathrm{~d}$ mesh is an array with regularised interval spacing, with the rows representing $\mathrm{x}$-coordinate and the columns representing y-coordinate. The elements of the array correspond to scaled z- coordinate. Up to this point a list of 3D vertices is present. These represent the distribution of false height and will be used for later for the VR display. 


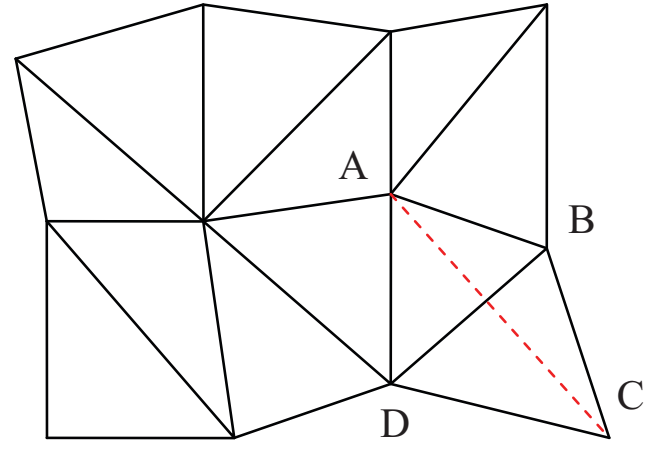

(a)

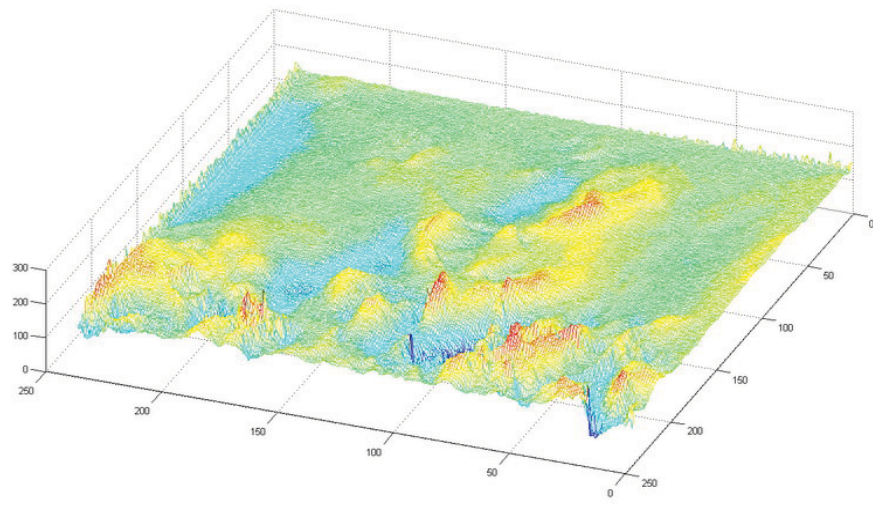

(b)

Figure 4 Triangulation of false height map: (a) Topology of the false height map; (b) 3D mesh of the false height map

Furthermore, to give a realistic surface finish in the $3 \mathrm{D}$ rendering, the surface texture of the painting may be superimposed onto the false height. For this purpose, it is not sufficient to merely use the previously calculated vertices, as both vertices and triangles are now required. It is worth noting that each vertex comes from structured 2.5D image and therefore has the connection relationship with its neighboring vertices. By using this topological relationship, the range surface can be constructed by simple triangulation. The process is as follows: ABCD are four adjacent range points in the range image. They can be connected into 2 triangles. From Figure 4(a) it can be seen that there are two possible ways to form two triangles by combining those four points: $\mathrm{ABD} \& \mathrm{BCD}$ or $\mathrm{ABC} \& \mathrm{ACD}$. Our procedure is to compute the lengths of the two diagonals $\mathrm{AC}$ and $\mathrm{BD}$ and to compare them. If $\mathrm{BD}$ is shorter, the triangle with the $\mathrm{ABD}$ and $\mathrm{BCD}$ combination would be used. Figure 4(b) shows the 3D mesh created by this strategy.

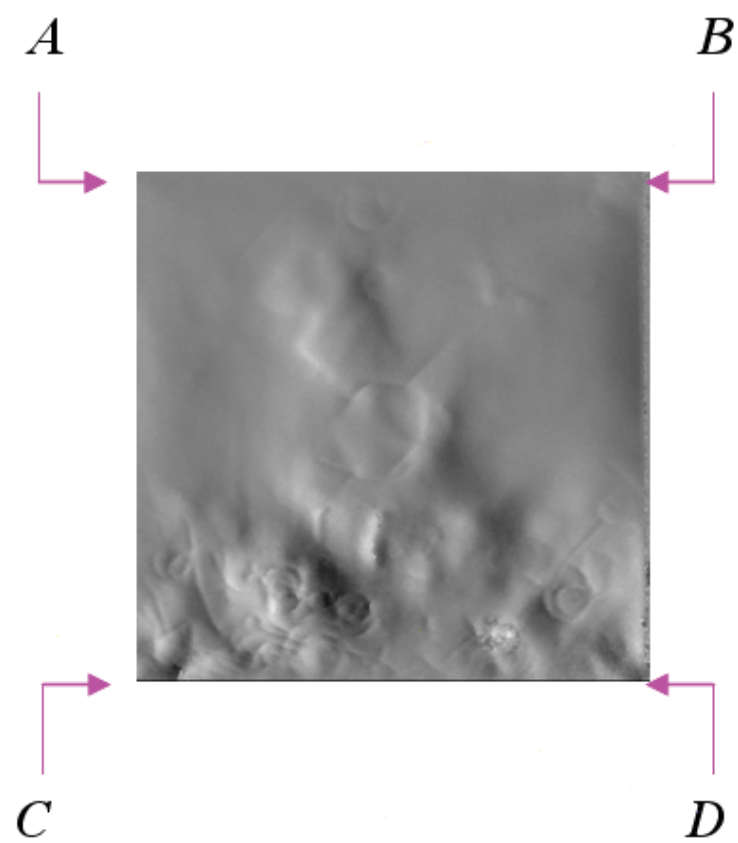

(a)

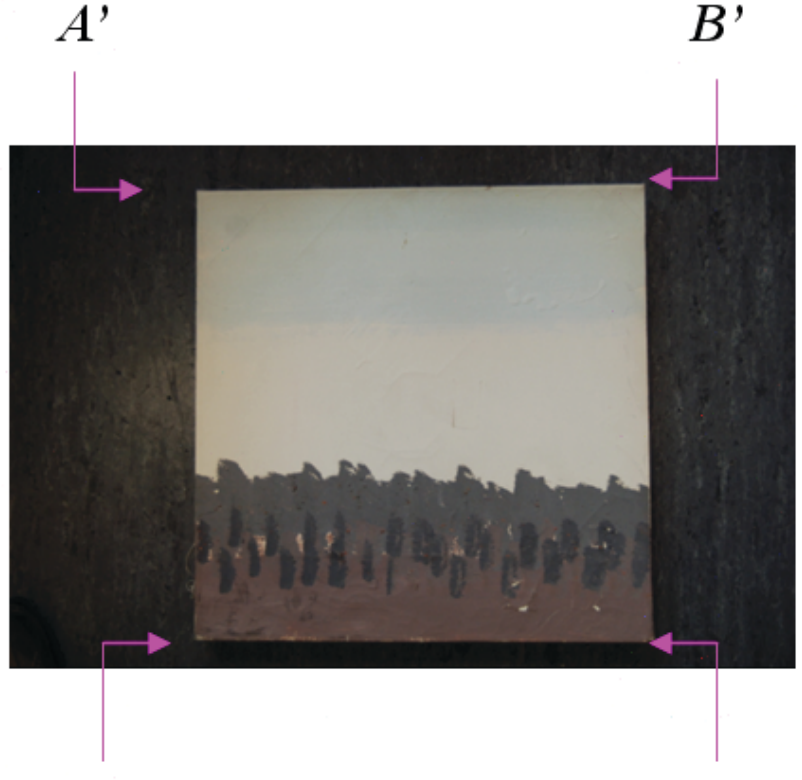

(b)

Figure 5. 2D homography calculation. (a) is the shearography displacement gradient input data and (b) is the colour photograph of the object under investigation

The following paragraph describes the procedure to combine the texture map with the false height map. $M$ is defined as the false height map and $M^{\prime}$ as the texture map. As shown in Figure 5, the coordinates of four corner points $(A, B, C, D)$ 
in the $M$ can be calculated. The corresponded texture points $\left(A^{\prime}, B^{\prime}, C^{\prime}, D^{\prime}\right)$ in $M^{\prime}$ can be detected using an edge extraction method using image processing. Then the homography matrix $\mathbf{H}$ between false height plane $M$ and texture plane $M^{\prime}$ can be determined using DLT method $\left.{ }^{10}\right]$. Therefore, for an arbitrary point $X$ in $M$, its corresponding texture point $X^{\prime}$ in $M^{\prime}$ by $X^{\prime}=\mathbf{H} X$, where $\mathbf{H}$ is a $3 \times 3$ non-singular matrix, can be calculated.

The virtual reality model calculated using these techniques is saved as a VRML format file for display. VRML is an ASCII format and a simple VRML file contains three parts. The first part is the format header which contains the information about the mesh. The second part is the list of vertices and triangles. The third part of the file is the pixel coordinates of the 3D mesh and 2D texture image. VRML viewers are freely downloadable from the internet, allowing VRML file users in remote locations to interact with the data.

\section{RESULTS}

\subsection{Sample Construction}

Following traditional practice, artists' canvas was tightly stretched on a wooden expandable stretcher, with mitred corners and metal turnbuckle expansion systems. The canvas was sized twice with rabbit-skin glue solution (in water, applied by brush), allowed to dry, and then primed with a white chalk glue ground applied by brush in two layers, allowing overnight drying. Various materials were applied to the ground layer as part of a test to detect under-painting, impasto and discontinuities. White paint in oil/alkyd medium mixed with dry pigments was applied in simple layers to create an image, including some isolated areas of thicker paint in the foreground. The painting was put into an oven at $55 \% \mathrm{RH}$ and $60^{\circ} \mathrm{C}$ for 1 month to thermally change the paint layer. Thermal ageing accelerates reactions in the paint film, here we have assumed by a factor of 2 for every $5^{\circ} \mathrm{C}$ increase in temperature above $20^{\circ} \mathrm{C}$, nominally 64 times at $50^{\circ} \mathrm{C}$ and 250 times at $60^{\circ} \mathrm{C}$. The increased temperature also increases the rate of diffusion of fatty acid plasticizer by an unknown amount. By these means it was possible to produce samples with the visco-elastic properties of a naturally aged paint, twenty years-old or more. Degradation of the canvas is much less advanced than that of the paint film since unlike many canvas paintings it has not been exposed to acid hydrolysis from a polluted atmosphere (sulphur dioxide). The samples were aged further in order to increase brittleness of the paint. In this second ageing process the oven was set at $60^{\circ} \mathrm{C}$ and $55 \%$ RH for six weeks. Afterwards, the canvas was re-tightened but the paint did not crack. Impact cracks were then created to simulate typical damage. This proved to be an excellent reproduction of the crack patterns seen on real aged paintings.

\subsection{Measurement procedure}

The displacement gradient was measured using the shearography instrument described in Section 2.2. This generated the displacement gradient map shown in Figure 5(a). The procedure for preparing the virtual reality file is described in detail in Section 3. The VRML file generated can be investigated with standard VRML interfaces, such as the Octaga interface shown in Figure 6.

The virtual reality procedures described are for 3D display in an interactive environment and it is therefore difficult to give a full impression in a printed manuscript. Supporting files and instructions for viewing are available at [3]. 


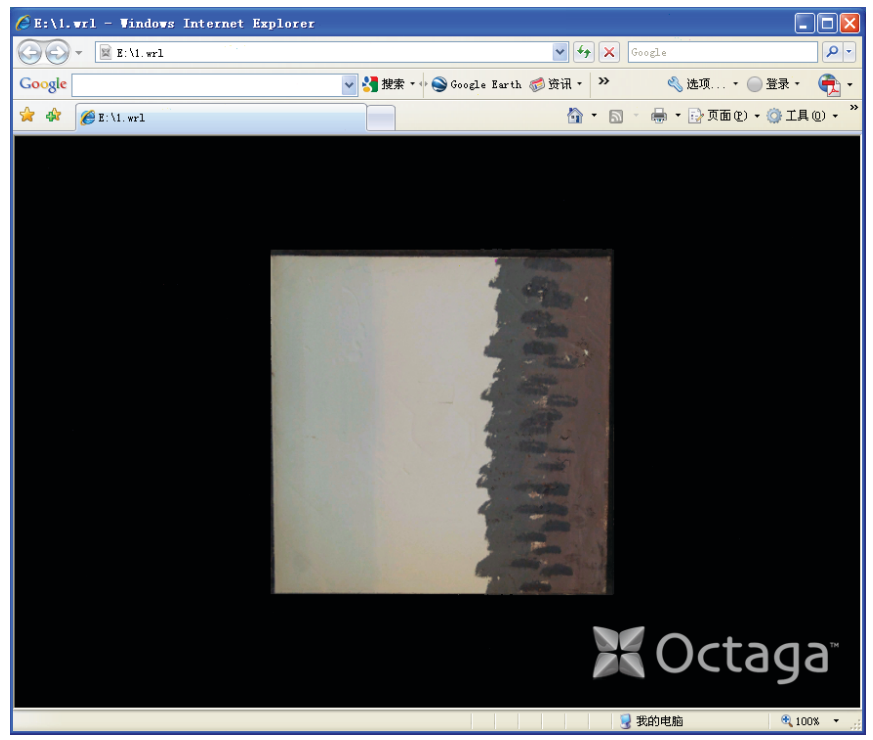

(a)

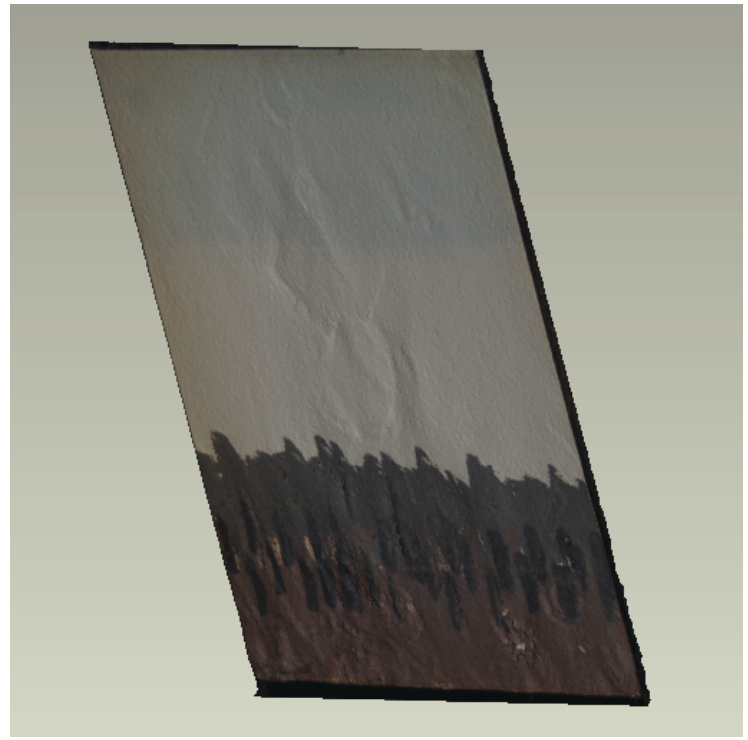

(b)

Figure 6. 2.5D virtual reality model of shearography strain displacement gradient data using the Octaga VRML interface to display the results. (a) shows the user interface and (b) shows a selected object viewing angle which gives some impression of the false height parameter.

\section{DISCUSSION}

The shearography displacement gradient measurement of the painting (Figure 5(a)) shows that a number of defects can be located from the phase map. This includes a larger circular defect in the centre of the sample painting. The damage location with be consistent with the sample painting vibrating at its first natural frequency during transit and impacting on the inside of the travel case. Further defects in the lower part of the painting were introduced during the sample manufacture and are due to variations in painting thickness and cracks introduced during the aging process. Overall shearography is an effective structural diagnostics tool for artwork with an ability to locate small defects. The complex construction and materials of artwork often result in complex displacement gradient data, which can be difficult to interpret from a conservation point of view.

As the shearography phase map does not contain object colour information it is difficult to determine the location of defects with respect to features in the painting. Figure 6(b) shows how the same defects are represented using the virtual reality viewer. The effect is rather like a raked light photograph, with the shadows formed by the surface height differences assisting the conservator to locate the defects. It should be noted that in a conventional raked light photograph the shadows are formed by real small differences in surface height, rather than the shadows due to false height in the virtual reality model.

The virtual reality model also has the advantage that the user can interact with the artwork remotely, rotating and zooming in or out from the artwork to assist in the study of the defects. Further virtual reality applications for structural diagnostics can be envisaged, including overlaying data from a range of different structural diagnostics instrumentation and for presenting results at conferences and workshops.

\section{CONCLUSIONS}

The authors have successfully demonstrated that false height in virtual reality can be used for the display of shearography displacement gradient maps. The technique allows the user to view both the displacement gradient measurements and the original colours of the painting. 


\section{ACKNOWLEDGEMENTS}

The construction of the shearography sensor was supported by the European Union funded MULTI-ENCODE (Multifunctional Encoding System for the Assessment of Movable Cultural Heritage) FP6 Project (006427 SSPI).

\section{REFERENCES}

[1] Eds. Di Marcello, S., Virgili, V., Roxas, S. A., Miraglia, F., TECHA 2008, International Forum for Technologies Exploitation for the Cultural Heritage Advancement, Rome, Italy (2008).

[2] Fotakis, C., Anglos, D., Zafiropulos, V., Georgiou, S. and Tornari, V., [Lasers in the preservation of cultural heritage], CRC Press, Boca Raton (2006).

[3] Weblink to supporting virtual reality files: http://www.lr.tudelft.nl/live/pagina.jsp?id=4712dd08-11fc-4fec-810e1522ae7ac893\&lang=en

[4] Steinchen, W., and Yang, L., [Digital shearography], SPIE Press, Bellingham (2001).

[5] Creath, K., "Temporal phase measurement methods", Robinson, D. W., and Reid, G. T., editors. In: [Interferogram analysis, digital fringe measurement methods], Institute of Physics, Bristol, pp. 94-140 (1993)

[6] Ghiglia, D. C., and Pritt, M. D., [Two-Dimensional Phase Unwrapping], J. Wiley, New York (1998)

[7] Brown, M. Z., Burschka, D., and Hager, G. D., "Advances in computational stereo", IEEE Trans. on Pattern Analysis and Machine Intelligence, 25(8), pp. 993-1008 (2003)

[8] Batlle, J., Mouaddib, E., and Salvi, J., "Recent progress in coded structured light as a technique to solve the correspondence problem: a survey", Pattern Recognition, 31(7), pp. 963-982 (1998)

[9] Salvi, J., Pages, J. and Batlle, J., "Pattern codification strategies in structured light systems", Pattern Recognition, 37(4), pp. 27-849 (2004)

[10] Richard, H., Andrew. Z., [Multiple view geometry in computer vision], Cambridge University Press, Second Edition, 88-90 (2003) 